Acta Theriologica $36(3-4): 315-328,1991$.

PL ISSN $0001-7051$

\title{
Kilometric index as biological indicator for monitoring forest roe deer populations
}

\author{
Jean-Paul VINCENT, Jean-Michel GAILLARD* and Eric BIDEAU
}

\begin{abstract}
Vincent J.-P., Gaillard J.-M. and Bideau E. 1991. Kilometric index as biological indicator for monitoring forest roe deer populations. Acta theriol. 36: $315-328$.

During a long term study (10 years) of an increasing forest roe deer Capreolus capreolus (Linnaeus, 1758) population, the reliability of a kilometric index for monitoring population size was tested. Kilometric index was defined by the ratio "number of observed animals/number of kilometers" of standardized transect. Three indexes were constructed: one for each transect, one for a transect set and one for all transects performed during a year. Actual population size was estimated each year by capture - mark - recapture. A modification of Petersen - Lincoln Index allowing long-term use was applied as the reference method. The relationship between annual kilometric index and population size estimation was assessed by calibrating index using linear and logistic regression. Moreover, we looked for different population levels using analyses of variance applied on the different indexes. Although high correlation was obtained between annual kilometric index and reference method, calibration equation led to unreliable population schedule. This demonstrated differential sensitivity of kilometric index according to population levels. Three population levels were discriminated by analyses of variance: a low population level during the four first years, a transition level corresponding to the fifth year and a high population level during the five last years. Indexes defined on a transect set (called "photo") appeared as the more informative. Such a study showed that to look for differential population levels from abundance indexes (bioindicator conception) is more pertinent than to establish conversion equations (calibration conception) in order to monitor forest roe deer population.
\end{abstract}

IRGM, INRA, BP 27, 31326 Castanet-Tolosan Cedex, France; *URA 243, UCB Lyon 1, 43 Bd du 11 novembre 1918, 69622 Villeurbanne Cedex, France

Key words: monitoring population size, Capreolus capreolus

\section{Introduction}

Although intensive research has been made since more than half a century into the design and development of census methods (Leopold 1933, Moen 1973, Caughley 1977a, Seber 1982), all the problems have not been solved. Size estimation of ungulate populations is time- and money-consuming. Its accuracy and reliability are limited. In the roe deer Capreolus capreolus (Linnaeus, 1758) it is still more difficult due to the high density of forest biotope in which these animals are living, and to the social structure of the species which leads to few groupings (Vincent et al. 1983, Bideau et al. 1983).

Roe deer census attempts have been numerous (Strandgaard 1972, Pucek et al. 1975, Boisaubert et al. 1979, Zejda 1984, Ratcliffe and Rowe 1985, Denis 1985, Staines and Ratcliffe 1987) but none has led to satisfactory results except the capture - mark - recapture 
methods (C.M.R.) when more than two third of individuals are marked (Strandgaard 1972, Gaillard et al. 1986).

Thus, approaches using index have been developed to replace the census method (Dice 1941, Eberhardt 1982, Eberhardt and Simmons 1987). The most important step in this kind of approach consists in the strict index calibration and study of its evolution compared to the corresponding biological phenomenon. After that, a mathematical model is used to translate the index into absolute numbers. A lot of trials have been performed (Caughley 1974, 1977b - for aerial census, Zejda 1984, 1985, Burnham et al. 1980 - for counts along transects, Neff 1968, White and Eberhardt 1980, Putman 1984 - for pellet counts).

With the aim of monitoring with accuracy forest roe deer populations, we chose an index already known for its efficiency in ungulate populations. Up to now, aerial census and line transects were the most successful methods, so that line transect can be considered as an absolute census method (Burnham et al. 1980). However, aerial census is not possible in forest because of the density cover and of its high cost. If line transects have already been used in roe deer populations (Gaillard 1988), the generalization of this method is limited for forest roe deer populations as it is necessary to measure the distance between the observer and the animals and to observe at least forty to sixty animals to get reliable results. Using the same sampling principle as the line transect, namely the line sampling, we chose to test a kilometric index (IK). The first investigation was done in populations with a density ranging between 15 and 20 animals/100 ha (Vincent et al. 1979, Vincent and Bideau 1982). In this paper, this index will be tested on a more large range of animal numbers, representative of the typical roe deer population growth from low density (less than 5 per 100 ha) to high density (more than 20 per 100 ha).

In these conditions, we tried to answer the two following questions: (1) Is it possible to get an absolute population density by means of a kilometric index along a large variation of roe deer population size? This implies to check kilometric index versus a reference method (capture - recapture method here). (2) Is it possible to use a kilometric index as an indicator of population trends? This implies to get reliable evaluation of the kilometric index sensitivity to population size fluctuations.

\section{Material and methods}

\section{Study area}

This study was carried out in a deciduous mixed forest, $40 \mathrm{~km}$ southwards Paris: Dourdan forest which is divided into 2 smaller units separated by the town of Dourdan (Fig. 1): northward state forest of St Arnoult-en-Yvelines (900 ha) and southward state forest of l'Ouye $(600 \mathrm{ha})$. The study took place in the St Arnoult forest. It is an open forest, just limited in its western part by A10 high-way ant Atlantique TGV railway.

In this area, the roe deer Capreolus capreolus (Linnaeus, 1758) is the most abundant species among ungulates, the red deer Cervus elaphus Linnaeus, 1758 and the wild boar Sus scrofa Linnaeus, 1758 staying there rarely and irregularly. Since 1980 , roe deer hunting has been completely stopped, leading to an increasing density from $5 / 100$ ha in 1980 to $25-30 / 100$ ha, 10 years later. Radio tracking monitoring allowed us to realize the very high rate of animal sedentariness. 


\section{Methods}

Individual marking represents the basic method. After capture with nets, roe deer were fitted with colars (Strandgaard 1967) or transmitters (Janeau et al. 1981). They were sexed, weighed and released after a rapid examination of their physical and parasitic condition.

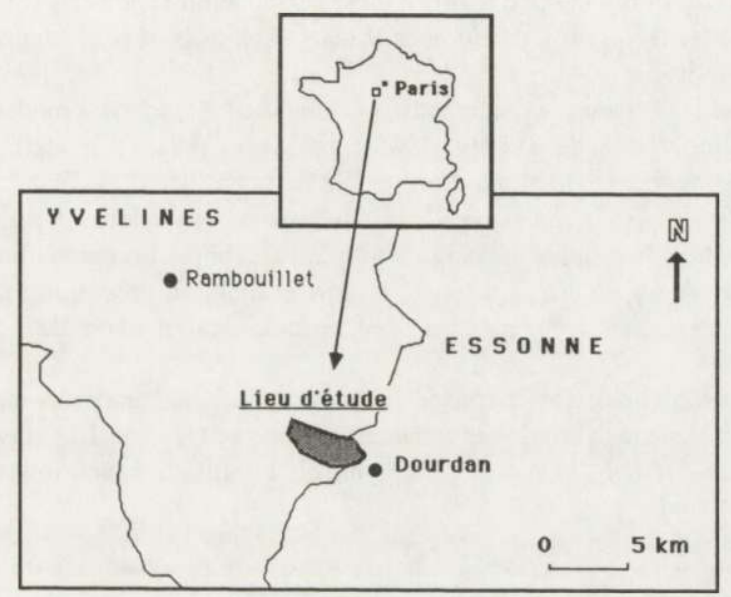

Fig. 1. Geographical situation of studied area.

Table 1. Main characteristics of transect standardized protocol.

\begin{tabular}{ll}
\hline Season & \multicolumn{1}{c}{ Winter, January - March } \\
\hline Timing & During the tree hours following sunrise or preceding sunset \\
Network & A network of fifteen transects overlays homogeneously the whole studied area \\
Repetitions & This set of transects is worked out from two to $n$ time between January and March \\
Index & Final Index kept down every years is calculated as the Mean of all "photo" Index (IKpi) \\
& For one "photo", IK = Sum (observations)/Sum (Kilometers) \\
\hline
\end{tabular}

D a t a c o 11 a ti o n. Population sampling was achieved by direct observation of the animals, during transects carried out on foot, according to a standard procedure (Vincent et al. 1979, Table 1).

Every year, two to six repetitions of the whole set transect were carried out. According to this method, three indexes were defined:

(1) $\mathrm{IKc}_{i}=$ number of contacts/Number of kilometers of transect " $i$ ".

For each set of fifteen transects (representing one census area and further calles "Photo"), a mean IK was determined:

(2) $\mathrm{IKp}_{i}=\mathrm{S} I K \mathrm{c}_{i} / 15$.

Likewise, the annual index was calculated as the mean of different "photos" ( 2 to 6$)$ perforemed during a given year:

(3) $\mathrm{IKa}_{i}=\mathrm{SIKp} /$ Number of "photos".

$\mathrm{D}$ a $\mathrm{t}$ a a $\mathrm{n}$ a $\mathrm{l} \mathrm{ys}$ i s. At the end of the transect period, number estimation was achieved using a C.M.R. method. Kilometric index calibration was based on this former estimation.

The total number of observations (contacts) was considered as a sample belonging to the population including a $M$ number of tagged animals. This is a drawing-putting back operation, and the binomial model can be used (Bailey 1951): 


$$
\hat{N}=\frac{M(n+1)}{m+1}
$$

where $M$ is the number of marked individuals during the census period, $n$ is the total number of observations (contacts), $m$ is the number of marked animals among these observations, $\hat{N}$ is the population size estimation.

However, the model (1) is only reliable within a closed population hypothesis (Seber 1982). If it is applied in the long term ( 10 years for this study), the number of marked animals $M$ is no longer known with a sufficient accuracy because of mortality.

To take into account this problem, two alternatives can be used: (1) to use a model allowing the occurrence of mortality for marked individuals (e.g. Jolly (1965)' and Seber (1965)'s model), (2) to correct $M$ rather empirically on the basis of marked animal monitoring. We first considered the first solution and obtained very large confidence intervals for population size estimations because of the lack of parsimony for such a model (Begon 1983). Moreover, based on radio-tracking monitoring and high observation pressure, a high fraction of dead animals was recovered. As no efficient model is now available to treat information on marked animals coming from both resighting and recovery, we used an empirical method taking into account all these informations.

It was considered that winter mortality occurred only after census, and that every tagged animal not observed for two successive years was dead. Variation in the number of tagged animals lies between two extreme points: the upper limit which considers all the non observed animals as still alive, and lower limit considering all of them as dead (Fig. 2).

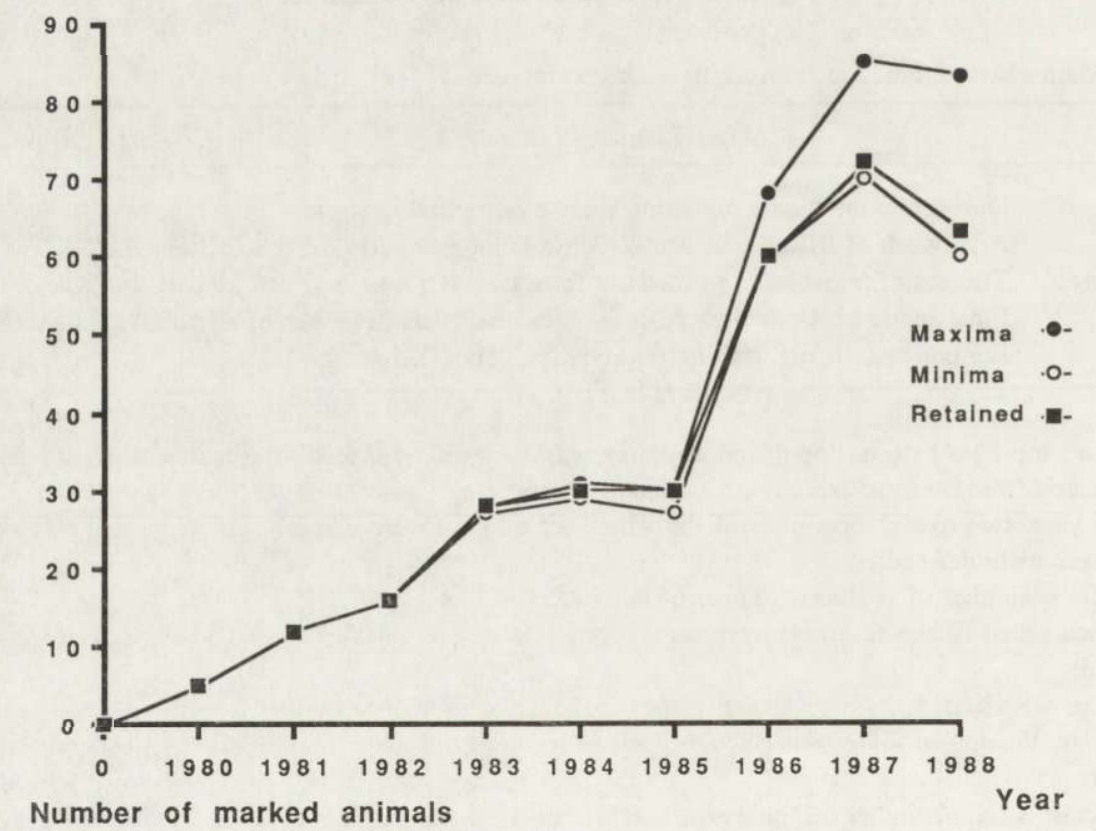

Fig. 2. Annual variation of the number of marked animals which are alive on the study area.

"Minima" represent the lower limit and are obtained by considering as dead all the marked animals not observed. "Maxima" represent the upper limit and are obtained by considering as alive all the marked animals not observed. "Retained" represent the estimations of the marked animal number used in the capture-recapture method. They are calculated by considering as dead all the marked animals not observed during two consecutive years. 
The second step in the data analysis consisted in studying the IK variation on the one hand, and the estimated number by means of C.M.R. method on the other hand, in order to verify if these variations comply with roe deer population growth.

The most simple way to describe population growth is to use the following equation:

$$
N_{t}=N_{0} e^{a t}
$$

or, its linnear transformation,

$$
\operatorname{Ln} N_{t}=\operatorname{Ln} N_{0}+a t
$$

where $N_{t}$ is the population size at time $t, N_{0}$ is the population size at time $0, a$ is the natural rate of increase.

The linear regression (equation (3)) was used for modelling time variations both in IK and population size with time. According to the few observed points, we did not perform a residual analysis using the run test (Daniel 1978) to verify the random scattering of negative and positive residuals with time.

According to this very simple model, logistic regressions were achieved according to the model:

$$
N_{t}=\frac{a}{1+b e^{-c t}}
$$

where $N_{t}$ is the population size at time $t, a, b$ and $c$ are the model parameters.

This model describes population growth over a time period (e.g. Kingsland 1985). A particular attention was paid to the maximum annual rate of increase $\left(r_{\max }\right.$ in Caughley 1977a) corresponding to $c$ parameter in equation (4), in order to check the reliability of variations given by the model. To that end, we considered the results of simulations performed from demographic parameters of a roe deer population during settlement (Gaillard 1988).

The IK calibration was achieved by regression analysis of IK variations on the population size estimated with C.M.R.

Analysis of different variation sources (IK variance) was performed using the 3 former indexes: (1) between-year variability ( $\mathrm{IKa}_{i}$ variance) which shows the reliability of this method to distinguish several population-age levels, (2) between - "photo" variability (IKp $i$ variance for one " $a_{i}$ ") which measures the method repeatability over a time period, (3) between-transects variability ( $\mathrm{IKc}_{i}$ variance for one " $\mathrm{a}_{i}$ " and one " $\mathrm{p}_{i}$ ") which measures the homogeneousness of the animal spatial distribution (spatial repeatability of the method).

Analyses of variance (ANOVA 1 and 2) were used to test the possible effect of year and transect factors, "photos" giving the repetitions. The variance among these different classes was considered as equal and the IK variable as normal. The contrast method (Scheffé test) (Scheffé 1959) was used to determine the different population-size levels highlighted by IK variations.

\section{Results}

\section{Population size estimation by C.M.R. as reference method}

The annual population size estimation was estimated by capture - recapture and Kilometric Index $\left(\mathrm{IKa}_{i}\right)$ was obtained for each of the ten years studied (Table 2 and Fig. 3).

\section{Analysis of IK variations and population size estimations}

Linear regressions between respectively $\mathrm{Ln}$ (IK) with time and $\mathrm{Ln}$ (population size estimation) with time show a high correlation $(r=0.94, p<0.05$ and $r=0.86, p<0.05$, respectively) (Fig. 4 and 5). So, $90 \%$ of IK variability and $74 \%$ of population size estimation variability can be explained by time variations. 
Table 2. Annual population size estimation obtained from capture-recapture method and annual kilometric index $(\mathrm{IK})$ with coefficient of variation $(\mathrm{CV})$ during the long-term study of Dourdan roe deer population.

\begin{tabular}{cccc}
\hline Years & IK & (CV) & $\begin{array}{c}\text { Population size } \\
\text { estimation }\end{array}$ \\
\hline 1980 & 0.183 & $(38.9)$ & 63 \\
1981 & 0.262 & $(18.6)$ & 44 \\
1982 & 0.338 & $(1.40)$ & 73 \\
1983 & 0.284 & $(21.0)$ & 128 \\
1984 & 0.485 & $(16.6)$ & 87 \\
1985 & 1.033 & $(18.2)$ & 121 \\
1986 & 1.270 & $(33.9)$ & 138 \\
1987 & 1.388 & $(13.4)$ & 146 \\
1988 & 1.383 & $(11.4)$ & 148 \\
1989 & 1.268 & $(16.1)$ & 146 \\
\hline
\end{tabular}

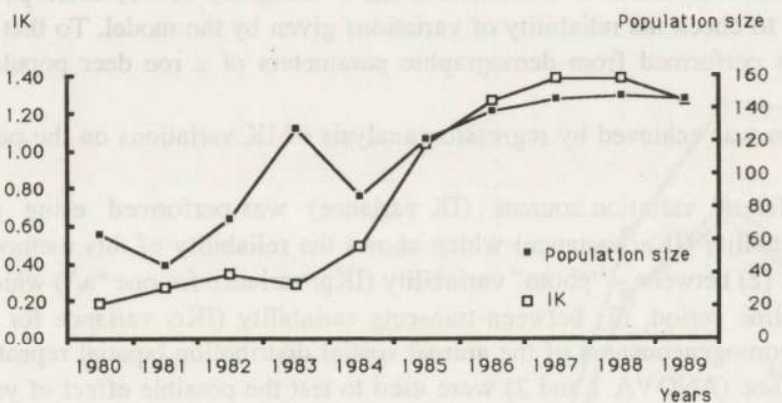

Fig. 3. Roe deer population size trends over time as measured by both a capture-recapture reference method (population size) and the annual kilometric index (IK).

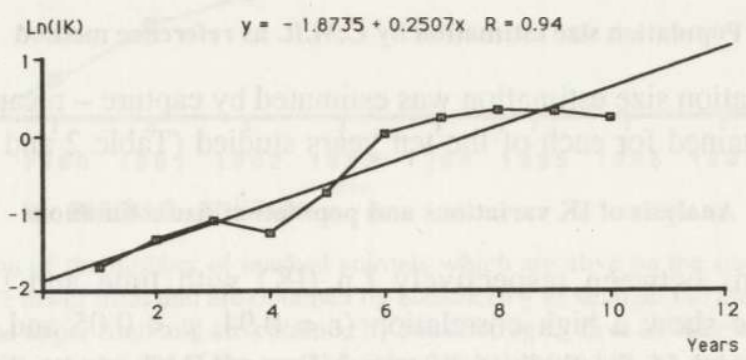

Fig. 4. Linear regression of annual kilometric index on time for Dourdan roe deer population. 
From a statistical point of view, the simple linear regression model is appropriate to describe both population size estimation variations and IK variations over time. In that case, the annual rate of increase (parameter $a$ in equation (2)) is equivalent to the regression slope. Thus the annual rate of increase estimated from IK data is 0.255 and it equals to 0.121 from the C.M.R. estimations. Related annual multiplication rates (corresponding to $e^{a}$ value in equation (2) and equivalent to $N_{t}+1 / N_{t}$ ) are equal to 1.290 and 1.129 respectively, which are to some extent lower than the values expected for settling roe deer populations (1.45-1.50) (Gaillard 1988).

Population size stagnation after a 8 year period (Fig. 3) is certainly due to this underestimation, and the logistic model, which takes this phase into account, seems to be more appropriate biologically. In fact some biological data clearly indicate some modifications in population growth, such as decrease of home range size for adult males, weight of young and number of kid by adult female.

Logistic fittings by equation (4) give the following relations (Fig. 6 and 7):

$$
\begin{aligned}
\mathrm{IK} & =\frac{1.4356}{1+63.65 e^{-0.803 t}} & R^{2}=0.94 \\
E & =\frac{160.12}{1+3.35 e^{-0.400 t}} & R^{2}=0.83
\end{aligned}
$$

where $E$ is the population estimation, $R^{2}$ is the determination coefficient.

The corresponding annual maximum rates of increase are 0.803 and 0.4 for IK and population size estimations respectively. Converted into multiplication rate, these values give for roe deer populations a rate of increase equal to 2.23 (for IK) and 1.49 (for population size estimations).

The Leslie matrix model (Leslie 1945 modified by Usher 1972) with maximum demographic parameters for a roe deer population showed that such a population could not present a multiplication rate higher than $1.45-1.50$ (Gaillard 1988). In spite of its good fitness to data, the logistic model applied to IK variations over time is not biologically appropriate to describe roe deer population growth pattern.

\section{IK Calibration}

IK calibration against population size estimations using a C.M.R. method gives a significant linear relationship $(r=0.853, p<0.05)$ (Fig. 8):

$$
\mathrm{IK}=0.011 E-0.455
$$

where $E$ is the annual population size estimation.

Thus, almost $3 / 4$ of IK variability are explained by the estimated population size variations. The estimation obtained in 1983 seems to be unreliable, perhaps because of a bad data collation (lack of well-trained observers). In addition, the number of animals equipped by collar may have been estimated incorrectly. If this questionable point is ruled out, the equation exhibits few changes: 


$$
\mathrm{IK}=0.012 E-0.495
$$

where $E$ is the annual population size estimation but in such circumstances, more than $95 \%$ (96.4) of IK variability is explained by the population size variations.

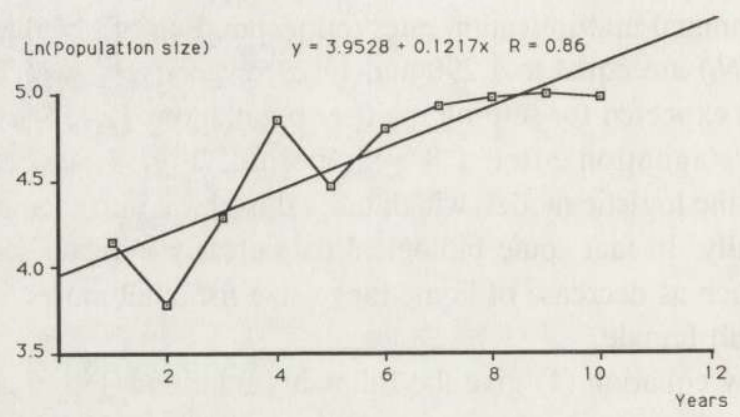

Fig. 5. Linear regression of annual population size estimated by capture-recapture method on time for Dourdan roe deer population.

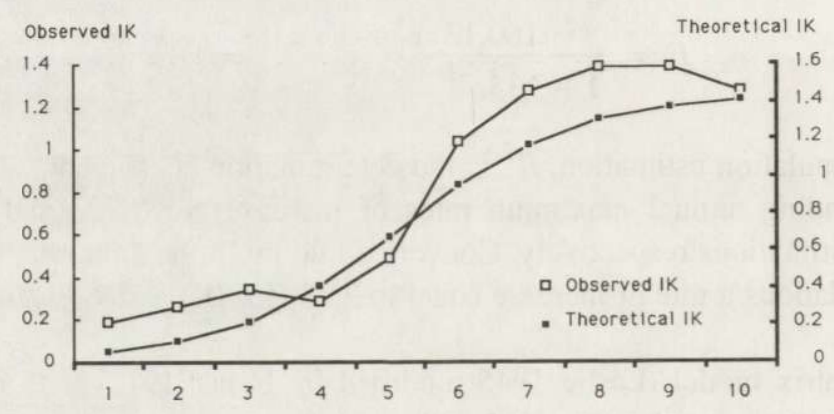

Fig. 6. Logistic regression of annual kilometric index on time for Dourdan roe deer population. "Observed IK" represents $\mathrm{IKa}_{i}$ calculated from field data. "Theoretical IK" represents the IK value predicted by logistic curve.

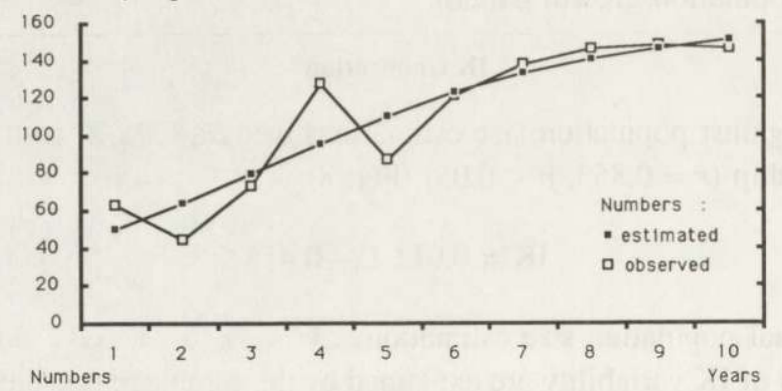

Fïg. 7. Logistic regression of annual population size estimated by capture-recapture method on time for Dourdan roe deer population. "Observed population size" represents the estimation obtained by capture-recapture method application. "Theoretical population size" represents the population size values predicted by logistic curve. 


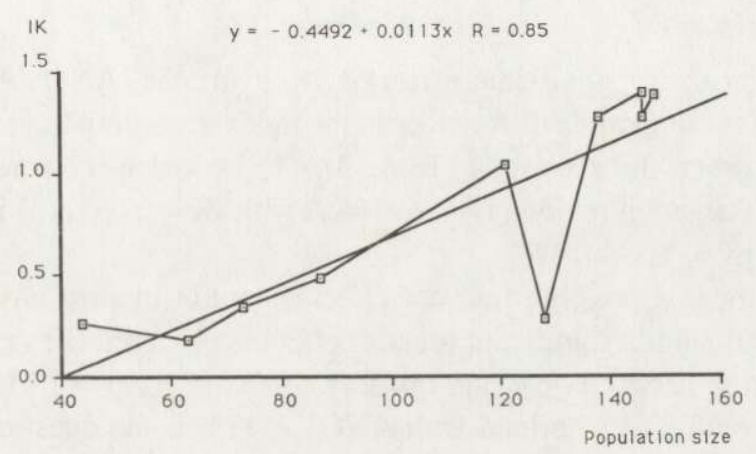

Fig. 8. Linear regression of annual kilometric index (IK) on annual population size estimated by capture-recapture method for Dourdan population.

Table 3. Contrast tests applied on the year effect analysis of $\mathrm{IKc}_{i}$ variable. A homogenous group is recognized by no significant inter-annual difference inside the group and significant inter-annual difference outside the group. So, 1980, 1981, 1982 and 1983 form a homogeneous group opposed to $1986,1987,1988$ and 1989 homogeneous group. 1984 and 1985 appear as intermediate. ns - not significant difference $(p>0.05)$ * - significant difference $(p<0.05)$.

\begin{tabular}{|c|c|c|c|c|c|c|c|c|c|c|}
\hline Years & 1980 & 1981 & 1982 & 1983 & 1984 & 1985 & 1986 & 1987 & 1988 & 1989 \\
\hline 1980 & - & ns & ns & $\mathrm{ns}$ & * & $*$ & $*$ & $*$ & $*$ & $*$ \\
\hline 1981 & & - & ns & ns & * & $*$ & $*$ & $*$ & $*$ & $*$ \\
\hline 1982 & & & • & ns & ns & $*$ & $*$ & $*$ & $*$ & * \\
\hline 1983 & & & & - & $*$ & * & * & $*$ & $*$ & * \\
\hline 1984 & & & & & - & $*$ & $*$ & $*$ & $*$ & * \\
\hline 1985 & & & & & & • & ns & * & $*$ & ns \\
\hline 1986 & & & & & & & - & ns & $\mathrm{ns}$ & ns \\
\hline 1987 & & & & & & & & - & $\mathrm{ns}$ & ns \\
\hline 1988 & & & & & & & & & - & ns \\
\hline 1989 & & & & & & & & & & • \\
\hline
\end{tabular}

Table 4. Contrast test applied on the year effect analysis of IKpi variable (cf. Table 3 for explanation).

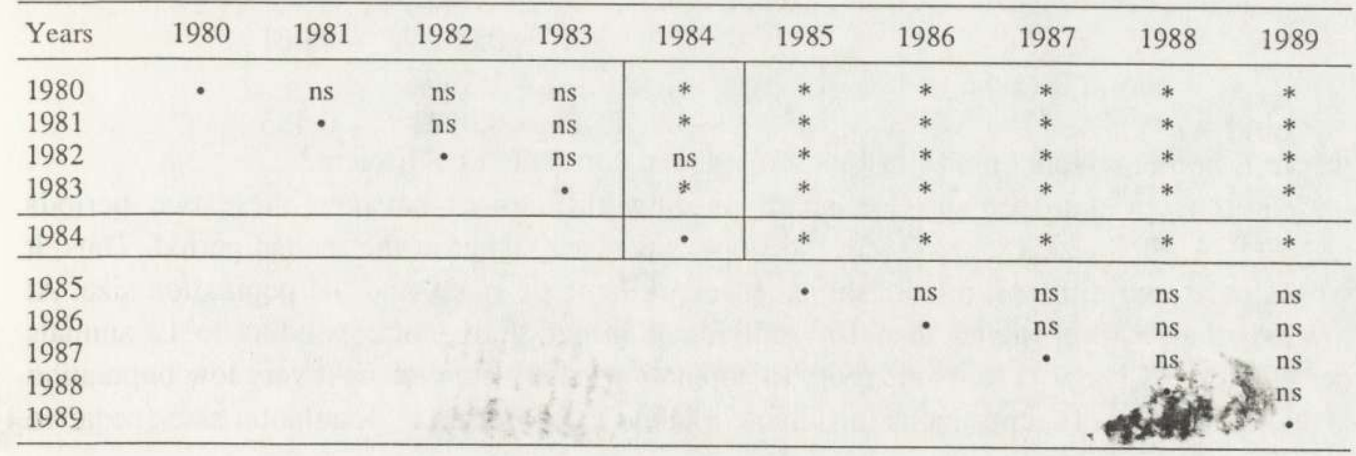




\section{IK as an indicator}

The year factor has a very significant effect on $\mathrm{IKc}_{i}$ variable (ANOVA $1 ; \mathrm{F}_{9,434}=35.61$; $p<0.0001)$. This points out marked differences in the index according to years, and the Scheffé test discriminates between three periods (Table 3): (1) Low density period (1980 to 1983) with IK $=0.241$, (2) Transition period (1984 to 1985) with IK $=0.631$, (3) High density period (1986 to 1989) with $\mathrm{IK}=1.334$

Taking into account a possible transect effect does not modify anything (ANOVA 2; $\left.\mathrm{F}_{9,220}=38.58 ; p<0.05\right)$, but a significant transect effect is observed $\left(\mathrm{F}_{10,220}=3.73 ; p<0.05\right)$ as well as an interaction between year and transect effects ( $\mathrm{F} 90,220=1.41 ; p<0.05)$.

The three former periods (as defined with ANOVA 1) become questionable. Once again, we tested the year factor, independently of the transect factor by means of ANOVA 1 on IKpi variable. Such an analysis pointed out 2 or 3 conditions for the studied population (Table 4): (1) Low density period (1980 to 1983) with IK $=0.240$, (2) Transition period (1984) with $\mathrm{IK}=0.497$, (3) High desity period (1985 to 1989) with $\mathrm{IK}=1.306$.

\section{Discussion}

Ungulate populations form with their environment a dynamic system (Caughley 1977a). From that point of view, ungulate population monitoring can be considered in two ways: (a) by appreciating the system as a whole, (b) by appresiating part of the system. The second way is mainly used by people involved in population management in which, most of the time, population size is looked for (Moen 1973, Caughley 1977a). Thus, abundance indexes are used as census methods after calibration. However, despite a rather good fitness observed during calibration trials, the perfect adequacy between index and population size cannot be assessed. This long term study of an increasing roe deer population demonstrated that the relationship between IK and population size, although very strong, can lead to useless parameters.

In fact, we can note that no IK value ranged between 0.5 and 1 (Table 2). Thus, it may be assumed that there are two different relations between IK and population size according to the density. Taking into account the best possible adjustment (ruling out the value of 1983) and only two periods for IK variation, adjustments become as follows:

$$
\begin{array}{ll}
\text { period } 1 \text { (1980 to 1984, except 1983) } & -r=0.781 \\
& \mathrm{IK}=0.0056 E-0.061 \\
\text { period } 2(1985 \text { to } 1989) & -r=0.947 \\
& \mathrm{IK}=0.0122 E-0.435
\end{array}
$$

where $E$ is the population size estimation, $r$ is the correlation coefficient.

Though our data set was too small to show differences between these two periods (ANCOVA, $\mathrm{F}_{1,5}=2.518 ; p>0.05$ ), the slope was twice higher in the second period. Thus, it would exist two different relationships between kilometric index and the population size. At low population level (lower than 100 individuals in our study, corresponding to 12 animals per $100 \mathrm{ha}$ ), IK shows a low sensitivity to population size fluctuations. At very low population level (close to 0 ), IK appears as unsuitable method to characterize population size (negative 
ordinate in calibration regression for period 1). This hyposensitivity of IK to population size variations prevents to obtain a strong relationship and only $60 \%$ of IK variability is explained by population size variability. At high population level (higher than 12 animals per $100 \mathrm{ha}$ ), IK shows a greater sensitivity which allows to obtain a strong relationship between IK variation and population size trends.

Because of this sensitivity fluctuation in IK response to population size trends, one IK value could be specific for low population level and the other could characterize high population level; the limit being roughly located near 100 animals (corresponding to 12 animals per $100 \mathrm{ha}$ ). This can explain bias (overestimate) of observed rate of increase. So, such an analysis shows that kilometric index calibration using a reference method (in that case Petersen-Lincoln method, corrected for long term application) is not suitable for a large scale

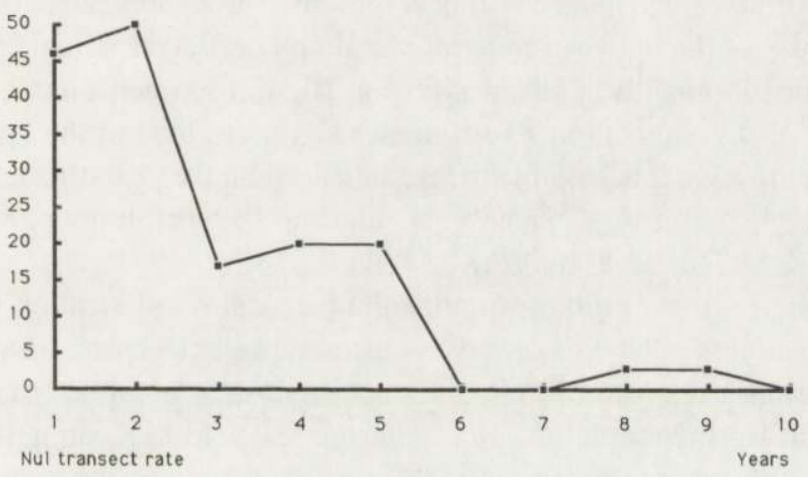

Fig. 9. Time variation of "nul transect rate" during the IK application on Dourdan roe deer population. "Nul transect rate" is defined by the number of realized transect leading to no contact with roe deer divided by the total number of realized transects for each year.

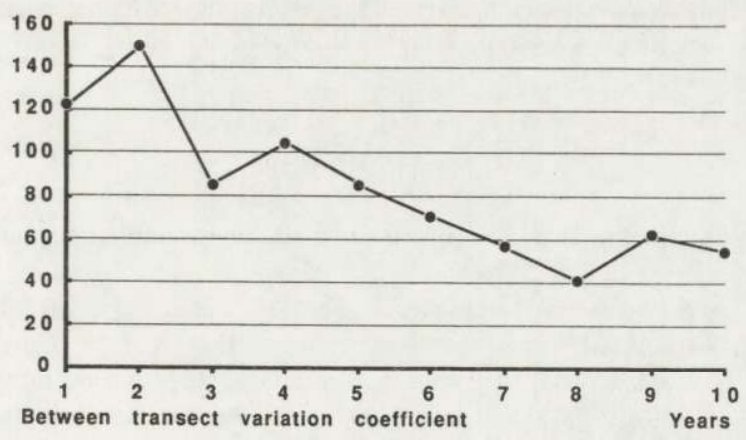

Fig. 10. Time variation of between-transect variation coefficient during the IK application on Dourdan roe deer population. "Between-transect variation coefficient" is defined by the standard deviation of realized transects within a "photo" divided by the mean value of these realized transects. 
of population sizes. Rather than trying to correct continuously (by successive calibration), it seems more reliable to determine some population levels.

As defined, kilometric index proved to be sensitive enough to distinguish between 3 successive population size levels, that is to say 3 conditions in the population-environment system of a growing roe deer population and such an information often appears as sufficient to monitor game populations. In that case, the analysis points out the interest of the "photo" variable which appears as the best compromise.

The lower level characterized by the transect $\left(\mathrm{IKc}_{i}\right)$ is indeed sensitive to other factors than the year; such as the transect or interaction between transect and year effects. It is not surprising if one considers that with small populations, the spatial distribution of roe deer is not uniform and that some areas are not yet occupied (numerous "nul transects" during the first fifth years, rare afterwards (Fig. 9). On the other hand, if the analysis is performed at the annual index level $\left(\mathrm{IKa}_{i}\right)$; the variability is canceled and it becomes difficult to appreciate the reliability and accuracy of the index. As the between-transect variation coefficient is higher than $80 \%$ with a small number of animals, and lower afterwards (Fig. 10), the between-transect variance might be used as indicator of the population size if analysis is performed at the "photo" level. So, according to the "photo" level it is possible to take into account the year effect, the repeatability of the index (many "photos" for the same year) and the between-transect variability (for a "photo", variance of $\mathrm{IKc}_{i}$ is used to build $\mathrm{IKp} i$ ).

All these analyses are based on the experimental protocol standarization which allows to minimize disturbing effects such as "observer performance" (Delorme 1989) or important meteorological variations. The use of "photo" level minimizes possible bias since effect of confusing factors (in Hurlbert opinion, 1984) introduces a lost of strength (variability is included in residual and reduces the $\mathrm{F}$ value), but no factor confusion (when variability is integrated into variance explained by the studied factor, here the year, and thus makes it possible to point out artefactually this factor).

Validity of $\mathrm{IKp} i$ as biological indicator allowing discrete monitoring (or in successive levels) of forest roe deer populations is thus receivable.

Acknowledgements: This study was successful because of the collaboration with personnel from Office National des Forêts. We thank Prs Legay and Pavé, Drs Lebreton and Pontier, and all the IRGM team research for their helpful and constructive comments.

\section{References}

Bailey N. T. J. 1951. On estimating the size of mobile populations from capture-recapture data. Biometrika 38: $293-306$

Begon M. 1983. Abuses of mathematical techniques in ecology: applications of Jolly's capture-recapture method. Oikos 40: $155-158$.

Bideau E., Vincent J. P., Quere J. P. and Angibault J. M. 1983. Occupation de l'espace chez le chevreuil (Capreolus capreolus L. 1789). I. Cas des mâles. Acta Oecologica, Oecologia applicata 4: 163-184.

Boisaubert B., Vassant J. and Delorme D. 1979. Contribution l'étude de la mise au point d'une méthode de recensement applicable l'espce chevreuil (Capreolus capreolus) vivant en milieu forestier. Bulletin Mensuel de l'Office National de la Chasse, No Sp. Sc. Tech.: $193-205$.

Burnham K. P., Andrson D. R. and Laake J. L. 1980. Estimation of density from line transect sampling of biological populations. Wildl. Monogr. 72: 1- 202. 
Caughley G. 1974. Bias in aerial survey. J. Wildl. Manage. 38: 921 - 933.

Caughley G. 1977a. Analysis of vertebrate populations. J. Wiley and Sons: $1-234$.

Caughley G. 1977b. Sampling in aerial survey. J. Wildl. Manage. 41: 605-615.

Clobert J. and Lebreton J. D. 1987. Recent models for mark-recapture and mark-resighting data. Biometrics 43: $1019-1022$.

Daniel W. W. 1978. Applied nonparametric statistics. Houghton Misslin Company, Boston: 1 - 503.

Delorme D. 1989. L'effet observateur: une source de biais lors de l'application de l'indice kilométrique d'abondance (IKA) pour le dénombrement de chevreuils (Capreolus capreolus). Gibier Faune Sauvage 6: $309-314$.

Denis M. 1985. Quelques méthodes pratiquées pour l'estimation de l'effectif d'une population de chevreuils (Capreolus capreolus L.). XVIIth IUGB Congress, Brussels: 979 - 989.

Dice L. R. 1941. Methods for estimating populations of mammals. J. Wildl. Manage. 5: 398 - 407.

Eberhardt L. L. 1982. Calibrating an index by using removal data. J. Wildl. Manage. 46: $734-740$.

Eberhardt L. L. and Simmons M. A. 1987. Calibrating populations indices by double sampling. J. Wildl. Manage. 51: $665-675$.

Gaillard J. M. 1988. Contribution la dynamique des populations de grands mammiféres: l'example du chevreuil (Capreolus capreolus). Unpublished Doctoral Thesis, Univ. Lyon.

Gaillard J. M., Boisaubert B., Boutin J. M. and Clobert J. 1986. L'estimation d'effectifs à partir de capture marquage - recapture: application au chevreuil (Capreolus capreolus). Gibier Faune Sauvage 3: 143-158.

Hurlbert S. H. 1984. Pseudoreplication and the design of ecological field experiments. Ecol. Monogr. 54: $187-211$

Kingsland S. E. 1985. Modeling nature. Episodes in the history of population ecology. University of Chicago Press, Chicago: $1-327$.

Janeau G., Quere J. P., Spitz F. and Vincent J. P. 1981. Etude par radiotracking des variations saisonniéres de l'étendue du domaine vital de cinq chevreuils (Capreolus capreolus). Biology of Behaviour 6: $291-304$.

Jolly G. M. 1965. Explicit estimates from capture-recapture data with both death and immigration-stochastic model. Biometrika 52: $225-247$.

Leopold A. 1933. Game management. Chas Scribner's Sons, New York: $1-481$.

Leslie P. H. 1945. On the use of matrices in population mathematics. Biometrika 33: $182-212$.

Moen A. N. 1973. Wildlife Ecology. W. H. Freeman and Co, San Francisco, California: $1-458$.

Neff D. J. 1968. The pellet-group count technique for big game trend, census, and distributions: a review. J. Wildl. Manage. 32: $597-614$.

Pucek Z., Bobek B., Łabudzki L., Miłkowski L. and Morow K. 1975. Estimates of density and number of ungulates. Polish ecol. Studies 1, 2: $121-136$.

Putman R. J. 1984. Facts from faeces. Mammal Review 14: 79 - 97.

Ratcliffe P. R. and Rowe J. J. 1985. A biological basis for managing red and roe deer in British commercial forests. XVIIth IUGB, Brussels: $917-925$.

Seber G. A. F. 1965. A note on the multiple-recapture census. Biometrika 52: 249 - 259.

Seber G. A. F. 1982. The estimation of animal abundance and related parameters. 2 th edition, Griffin, London: $1-654$.

Scheffé H. 1959. The analysis of variance. Wiley, New York: $1-477$.

Staines B. W. and Ratcliffe P. R. 1987. Estimating the abundance of red deer (Cervus elaphus L.) and roe deer (Capreolus capreolus L.) and their current status in Great Britain. Symp. zool. Soc. London 58: $131-152$

Strandgaard H. 1967. Reliability of the Petersen method tested on a roe deer population. J. Wildl. Manage. 31: $643-651$

Strandgaard H. 1972. The roe deer (Capreolus capreolus) population at Kälo and the factors regulating its size. Danish Rev. Game Biol. 7: 1 - 205.

Usher M. B. 1972. Developments in the Leslie matrix model. [In: Mathematicals models in ecology. J. N. R. Jeffers, ed.] : $29-60$. 
Vincent E. and Bideau E. 1982. Note sur la détermination d'un coefficient de visibilité en forêt: application au recensement du chevreuil. Acta Oecologica, Oecologia applicata 3: 257 - 262.

Vincent J. P., Bideau E. and Maire F. 1979. Vers une nouvelle méthode de recensement du chevreuil. Bulletin Mensuel de l'Office National de la Chasse, No Sp. Sc. Tech.: 207 - 226.

Vincent J. P., Bideau E., Quere J. P. and Angibault J. M. 1983. Occupation de l'espace chez le chevreuil (Capreolus capreolus L.). II. Cas des femelles. Acta Oecologica, Oecologia applicata 4: 379 - 389.

White G. C. and Eberhardt L. E. 1980. Statistical analysis of deer and elk pellet-group data. J. Wildl. Manage. 44: $121-131$.

Zejda J. 1984. Road strip transects for estimating field roe deer density. Folia zool. 33: 109 - 124.

Zejda J. 1985. Field transects for roe deer census. Folia zool. 34: 209-215.

Received 13 August 1990, revised 27 August 1991, accepted 27 August 1991. 